\title{
A case of pulmonary benign metastasizing leiomyoma: clinicopathologic, immunohistochemical, and PET/CT analyses
}

\author{
Qingguo Li ${ }^{1 \#}$, Youzhen $\mathrm{Wei}^{2 \#}$, Xuejun $\mathrm{Zhao}^{3}$, Chunling $\mathrm{Li}^{1}$, Shenglong Gao ${ }^{1}$, Bo Chen ${ }^{2}$, Shuliang Song ${ }^{2}$, \\ Qing $\mathrm{Gao}^{2}$, Chengbin $\mathrm{Ma}^{3}$, Qinchuan $\mathrm{Li}^{4}$ \\ ${ }^{1}$ Medical Image Department, Jinan People's Hospital, Jinan 271199, China; ${ }^{2}$ Research Center for Translational Medicine, Shanghai East Hospital, \\ Tongji University School of Medicine, Shanghai 200120, China; ${ }^{3}$ Gynaecology and Obstetrics, Changning Maternity and Infant Health Hospital, \\ Shanghai 200051, China; ${ }^{4}$ Department of Thoracic Surgery, Shanghai East Hospital, Tongji University School of Medicine, Shanghai 200120, China \\ \#These authors contributed equally to this work. \\ Correspondence to: Qinchuan Li. No.150 Jimo Road, Shanghai 200120, China. Email: doctqinchuanli@163.com.
}

\begin{abstract}
The pulmonary benign metastasizing leiomyoma (BML) is an extremely rare disease case, of which the pathogenesis was not elucidated. The accumulation of additional case reports and analyses is needed. A case of pulmonary BML is presented. A 48-year-old asymptomatic female patient that underwent a hysteromyomectomy 11 years ago was found to have abnormal shadows on a chest X-ray during a regular health check-up in 2012. Multiple nodular was observed in both lungs and uterine under computed tomography (CT) examination. Transbronchial lung biopsy surgery with a resection of the lesion was performed. The results revealed the pulmonary nodule was formed by benign smooth muscle cells which displayed very low mitotic activity. The samples showed highly positive signals for $\alpha$-smooth muscle actin ( $\alpha$ SMA), Desmin and Vimentin. Estrogen receptor (ER) and progesterone receptor (PR) were also immunoreactive positive. HMB-45, CD117 (C-kit) and S-100 were found to be negative with low Ki-67 index in the tumor cells. The pathological diagnosis was pulmonary BML. A total hysterectomy with adnexectomy were performed for the multiple uterine leiomyomas and pulmonary BML one week after diagnosis was made. From then on, the pulmonary nodules in both lungs were stable in 7 years postoperation, based on chest CT scan. When a solitary lung nodule is detected in a patient with a history of uterine leiomyoma, as an option, pulmonary BML, accurately diagnosing and proper treatment to has important implications for clinical outcome.
\end{abstract}

Keywords: Leiomyoma; benign lesion; lung; metastases

Submitted Jun 26, 2019. Accepted for publication Sep 28, 2019.

doi: $10.21037 /$ tcr.2019.10.02

View this article at: http://dx.doi.org/10.21037/tcr.2019.10.02

\section{Introduction}

Benign metastasizing leiomyoma (BML) is an extremely rare case, which often occurring in the lungs of women with a history of uterus leiomyoma (1). The pathogenesis of this disease was not elucidated. In most cases, there is a previous history of total hysterectomy or myomectomy for uterine leiomyoma. Generally, the lesions are asymptomatic, slowgrowing. And because of impercipient, they were usually misdiagnosed and underwent with improper treatment. To date, the origin of the tumor remains controversial, and the concept of metastasizing leiomyoma is still elusive. Some medical literature supports the concept that BML originates from an antecedent uterus leiomyoma. For more clinical support, the accumulation of additional case reports and analyses is needed.

\section{Case presentation}

A case of lung leiomyoma is reported, a 48-year-old female patient with a history of myomectomy for uterine 
leiomyoma 11 years ago. The patient was admitted for further examination of scattered shadows in both lungs that were found incidentally on the chest X-ray radiograph during a regular checkup in February 2012. A chest computerized tomography (CT) and positron emission tomography-computerized tomography (PETCT) were also performed. The patient was found to have a myomectomy in 2001, with a pathological diagnosis of a uterine smooth muscle tumor. During return visits, uterine leiomyoma was detected by B sonography, with maximum size of $3.7 \mathrm{~cm}$ in 2009, and $6.0 \mathrm{~cm}$ in 2012 (data not shown), without any treatments.

Multiple small solitary nodules with clear demarcations were detected in both lungs through CT (Aquilion 64, Toshiba Medical Systems, Tochigi, Japan) examinations. The diameter of maximum lesion from the left upper lobe was $3.0 \mathrm{~cm}$. No abnormal fluorodeoxyglucose were detected in the suspicious nodules under PET-CT (Discovery LS, GE Medical Systems Co Ltd., USA) examinations. The standard uptake value (SUV) value of these nodules was only around 1.7 (Figure 1) (SUV <2.0, benign lesion). The other clinical examination was unremarkable, results from routine laboratory examinations were also normal (data not shown). The diagnosis of suspected pulmonary metastasizing leiomyoma was drawn.

The patient underwent transbronchial lung biopsy of the lesion in March of 2012. The maximal lesion from the left upper lobe was resected for pathologic diagnosis. The cells from the samples were found to be benign in appearance and were fully differentiated with intraoperative frozen and postoperative pathological examination (Figure $2 A$ ). The cells showed spindle-like morphology and had abundant clear cytoplasm with distinct cell borders. The mitotic activity of the cells was low $(<0-1 / 10$ high-power fields), and had no cytological cellular atypia. No tumor cell necrosis or degeneration were noticed on the gross or microscopic examination (Figure 2A). No obvious changes were found in uterine tissue of leiomyoma from 2001 and 2012, and no malignant features were observed in these samples (Figure 2B,C).

Positive signal of immunostaining $\alpha$-smooth muscle actin $(\alpha \mathrm{SMA})$, Desmin, Vimentin, estrogen receptor alpha (ER $\alpha)$ and progesterone receptor (PR) (1:200; Long lsland Biotec. Shanghai, China) were detected in the immunostaining of examined pulmonary nodule tumor cells, compatible with tumor arising from the uterine smooth muscle. The spindle cells showed diffuse immunopositivity for aSMA
(Figure 3A,B,C), ER $\alpha$ (Figure 3D,E,F), PR (Figure 3G,H,I), but neither nuclear atypia nor mitotic activity were identified. The neural tissue marker S-100, lymphangioleiomyomatosis cell marker HMB-45 and CD117 (c-Kit) (1:200; Long lsland Biotec., Shanghai, China) were negative. The proliferation index defined by Ki-67 immunostaining (1:200; Long lsland Biotec., Shanghai, China) was very low, at approximately $1 \%$ (Figure 37,K,L). A diagnosis of BML was made based on clinical, radiological, histological features as well as immunophenotype of the lesion.

In consideration of that, the tumors would have changed their morphology to frank sarcoma if they had recurred several times, one week later, the patient accepted panhysterosalpingo-oophorectomy without any therapy except calcium supplement. The patient was discharged from the hospital without any early postoperative complications. Her current general condition was satisfactory without any radiological evidence of recurrent disease or distant metastases. Until now, there has been no recurrence and the patient remain asymptomatic. Besides before bilateral salpingo-oophorectomy (Figure 4A, $A^{\prime}$ ), the patient underwent the chest CT scan at 19 months (Figure 4B,B'), 28 months (this and thereafter, CT scan by Neviz64e, Shenyang Neusoft Medical System Co. Ltd. Shenyang, China) (Figure 4C, $\left.C^{\prime}\right), 48$ months (Figure 4D, D'), 60 months (Figure 4E, E'), and 77 months (Figure 4F,F') of postoperation. On repeat image measuring, the nodules showed regular morphology, well demarcated margin, without exudation nor infiltration, and remained unchanged in 7 years. The diameter of the big oval nodule in left lung, which homogeneous solid soft tissue shadow, was seemingly decreased, but not significant, simultaneously without obvious morphological change (Table 1). Liquefied necrotic sign, cavitary or cystic, has not been observed.

\section{Discussion}

In 1889 , Krische reported the first case in which fibromyomas of the uterus was found metastasized to many parts of the body. Later in 1939, Steiner et al. reported the first fibroleiomyoma case that showed benign appearance and transferred from the uterus to the lung (1). Several similar reports were published afterwards, and this disease was later renamed to 'benign metastasizing leiomyoma'. Pulmonary BML is an extremely rare disease in which tissue from a benign uterine leiomyoma is detected as a solitary nodule or as multiple nodules in lungs of the patients with a 


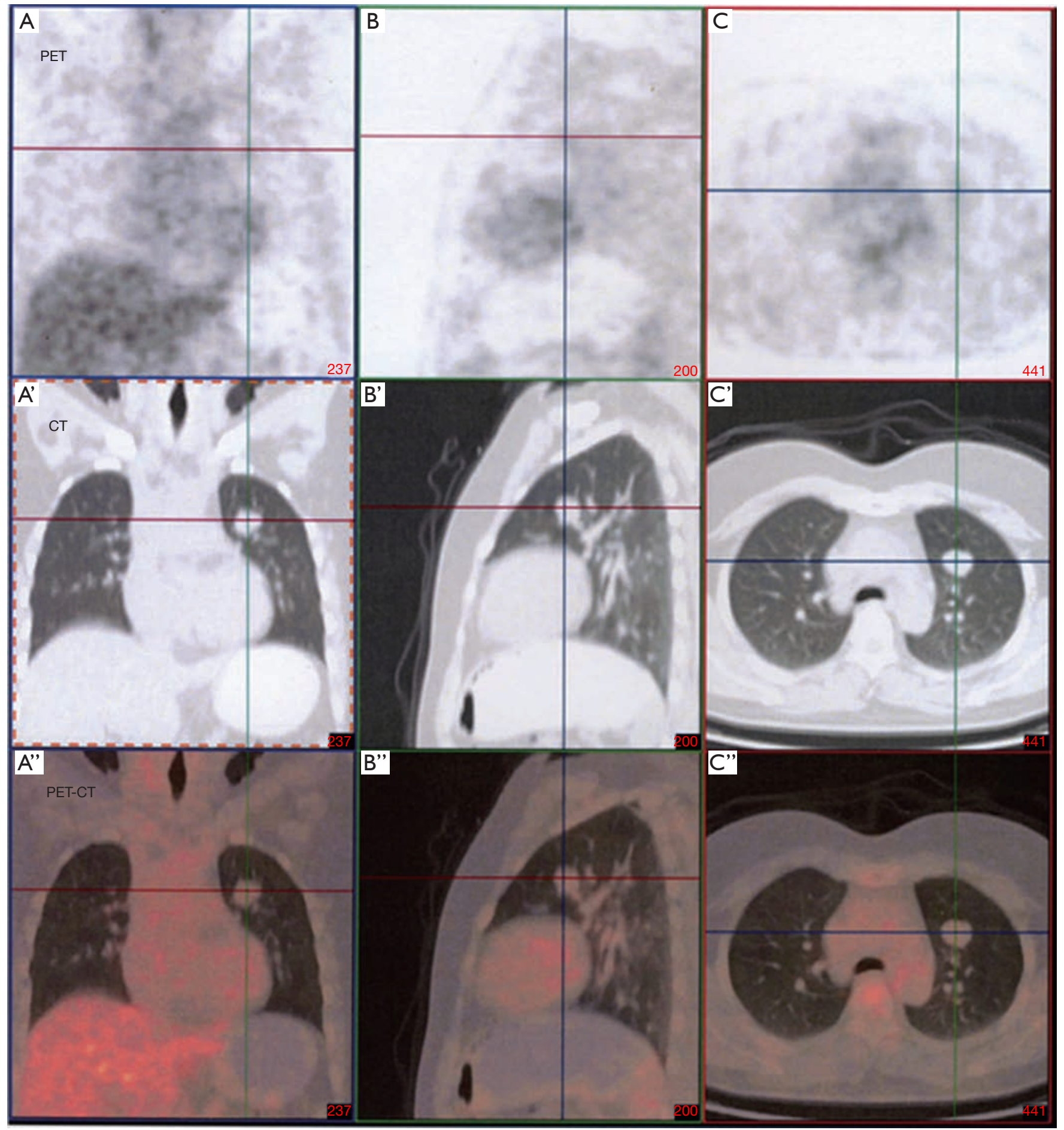

Figure 1 Chest Positron emission tomography-computerized tomography (PET-CT) scan in 2012. Images were presented and viewed in column A, coronal; column B, sagittal; column C, transaxial; and A", B”, C”, PET-CT fusion images. CT shows multiple nodules in both lungs, the diameters of maximum lesion (+) from the left upper lobe (A', B', C') was $3.0 \mathrm{~cm}$. PET did not show abnormal fluorodeoxyglucose uptake in suspicious nodules (+), the SUV value was about 1.7 (A, B, C, A", B", C”). 

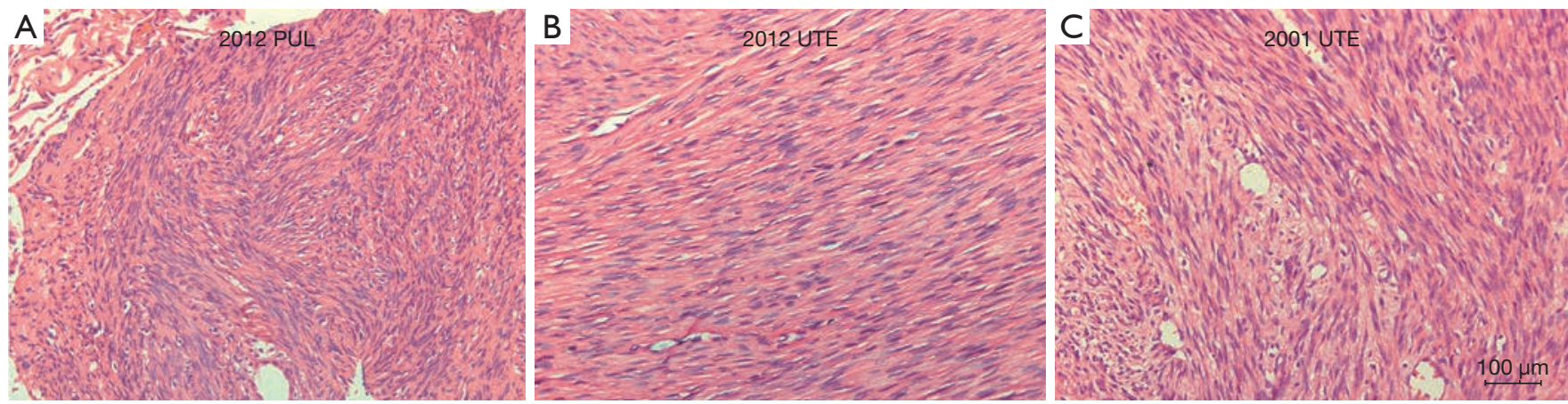

Figure 2 Histopathologic examination of the resected uterine and pulmonary leiomyoma showed a tumor consisting of well-differentiated spindle-shaped cells with intervening collagen (HE, ×200). Either uterine tissue of leiomyoma from 2001 and 2012 , or pulmonary needle biopsy showed spindle-shaped smooth muscle cells with non-atypical nuclei and eosinophilic cytoplasm proliferated in an anastomosing fascicular pattern. No malignant features were observed in these samples. (A) Pulmonary leiomyoma; (B) uterine leiomyoma in year 2012; (C) uterine leiomyoma in year 2001. UTE, uterine; PUL, pulmonary.

previous history of hysterectomy of uterine leiomyoma.

Majority of the pulmonary BML patients are asymptomatic, or non-specific, such as cough, dyspnea, and chest pain. Normally it is detected incidentally on routine chest imaging. It has been reported that the average age of patients with BML is 48 years old, and it takes 3 months to 26 years to detect nodule detection after hysterectomy (2). Although Tsunoda et al. (3) reported one case of BML of the lung complicated with primary lung cancer, all other cases reported experienced a benign process and had good progress.

The underling pathogenesis of BML is not clearly understood. It was reported that uterine leiomyoma is depended on hormones $(1,4)$. ER $\alpha$ and PR in case of BML usually have high expression, and drugs such as GnRH-a, progestin or ovariectomy can make the lesions regress or remain stabilized, suggesting that estrogen and progesterone may play an important role in the course of the pathogenesis.

Making the diagnosis of BML is very difficult. Confirming smooth muscle origin and the positivity of $\mathrm{ER} \alpha$ and $\mathrm{PR}$ via immunohistochemistry remains paramount to establishing the diagnosis. In this case, the cells from the biopsy samples showed spindle like shapes, and was found to have no mitotic activity or nuclear atypia. The examined cells showed strong aSMA signal, indicating the cells were smooth muscle origin. Moreover, the cells were negative for CD117, which excluded the possibility of extragastrointestinal stromal tumor. The absent of S100 in the cells, make it unlikely for the tumor to be neural origin.
Positive signal of ER $\alpha$ and PR suggests these tested cells were uterine smooth muscle cells.

At present, there is no existing consensus guideline for BML treatment. Conservative approach is normally suggested for this asymptomatic disease. Therapy regime of this disease include careful observation, surgical resection, hysterectomy and bilateral oophorectomy with antiestrogen management, progestins, aromatase inhibitor and or medical castration using luteinizing hormone-releasing hormone analogs (5). Treatment with oophorectomy or agents that decrease estrogen or progestins has resulted in tumor regression (6). Till today, surgery combined with hormone therapy is regarded as the best treatment of BML $(7,8)$. In pulmonary BML cases, radical surgical resection is conducted as the primary treatment. For unresectable cases, aromatase inhibitor (9), or long-acting GnRH analogs therapy are suggested as they are proved to suppress the endogenous gonadotropin secretion that is necessary for gonadal steroid production. According to Duncan et al. (10), ovariectomy or GnRH-a therapy after making diagnosis by transbronchial lung biopsy is one good choice of treatment, which minimizes the invasion, effective and safe. A standard strategy remains to be established for the treatment of this disease. But as the clinical course of BML varies among cases, specific treatment approaches should be considered $(11,12)$.

\section{Conclusions}

The incidence of BML is considerably low. However, it still 

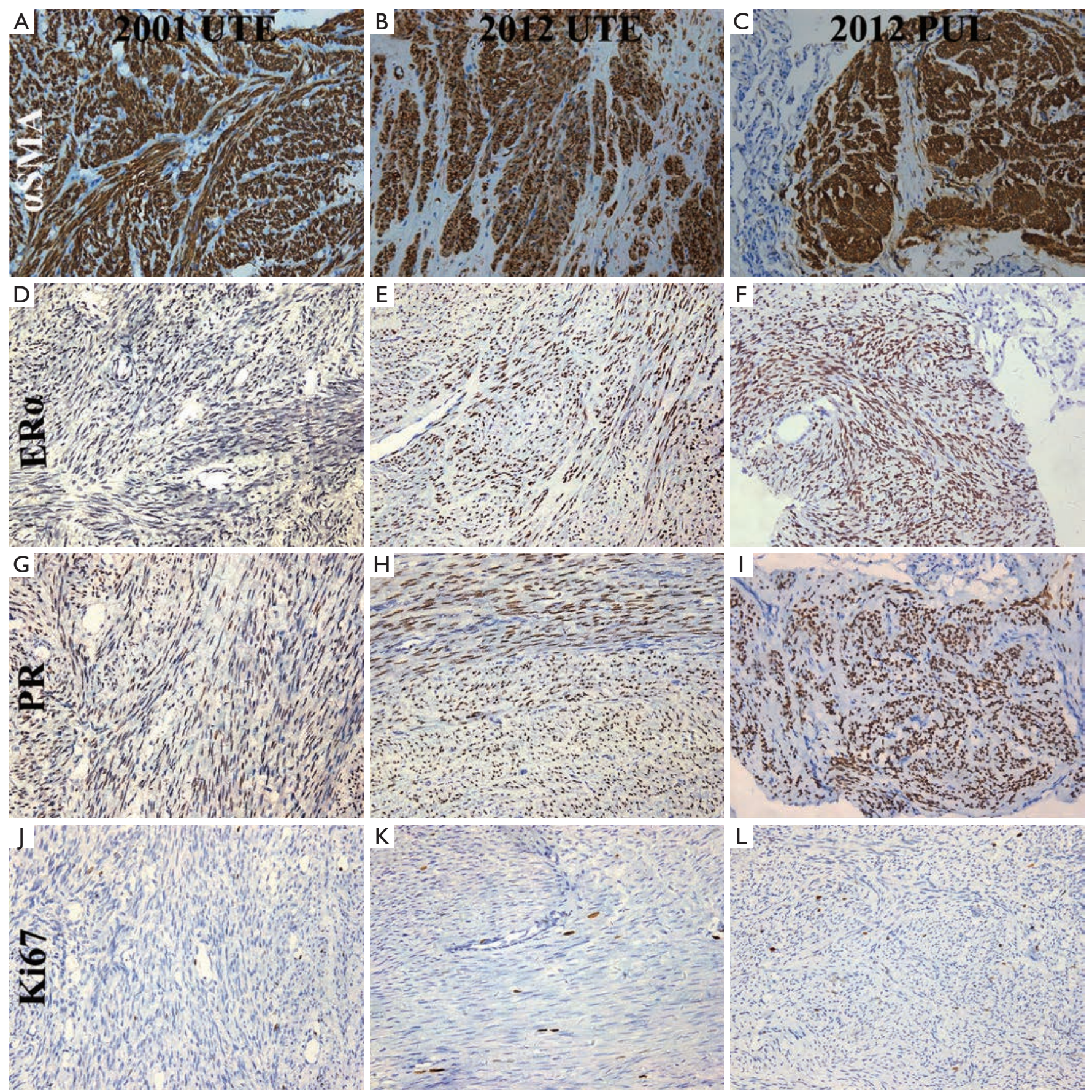

Figure 3 Immunohistochemistry of the uterine tumor and the lung nodule $(\times 200)$. (A,B,C) The tumor cells were diffusely positive for $\alpha$-smooth muscle actin ( $\alpha \mathrm{SMA}) ;(\mathrm{D}, \mathrm{E}, \mathrm{F})$ the nuclei showed positive staining for estrogen receptor alpha (ER $\alpha) ;(\mathrm{G}, \mathrm{H}, \mathrm{I})$ the nuclei staining was positive for progesterone receptor (PR); (J,K,L) immunostaining for Ki67. UTE, uterine; PUL, pulmonary. 

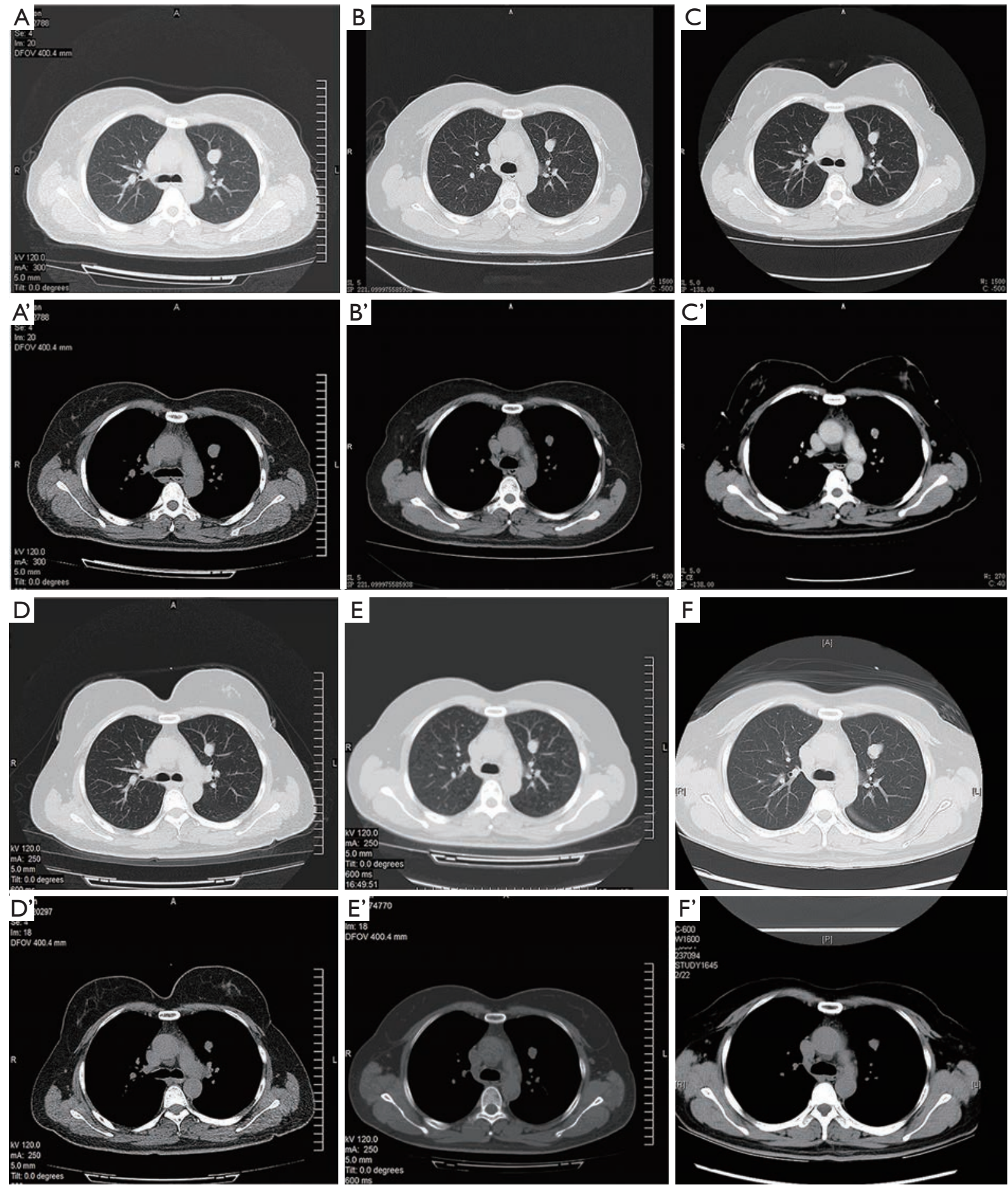

Figure 4 Chest CT scan showed multiple nodules in both lungs decreased. The CT scans were acquired before bilateral salpingooophorectomy (A, A') and postoperation at 19 (B, B'), 28 months (C, C'), 48 months (D, D'), 60 months (E, E'), and 77 months (F, F'). (A-F) Transaxial images of lung window, showed the nodules regular morphology, well demarcated margin, without exudation nor infiltration; (A'-F') soft tissue window, displayed an oval homogeneous solid soft tissue nodule, the decreasing of the diameter of the big nodule in left lung was not significant, nor obvious morphological change. And C' (contrast enhancement), the nodule was uniformly moderate enhancement. The number of pulmonary nodules in both lungs did not change. 
Table 1 The temporal variation of the big nodule in the left lung

\begin{tabular}{lcccccc}
\hline \multirow{2}{*}{ Variables } & \multicolumn{5}{c}{ Date } \\
\cline { 2 - 6 } & Feb 10, 2012 & Sep 14, 2013 & Jun 25, 2014 & Feb 29, 2016 & Mar 23, 2017 & Aug 14, 2018 \\
\hline TRS $(\mathrm{mm})$ & $20.65 \times 17.22$ & $18.84 \times 13.94$ & $18.37 \times 13.42$ & $19.93 \times 13.83$ & $19.24 \times 12.32$ & $19.69 \times 14.36$ \\
MPR $(\mathrm{mm})$ & No & $21.38 \times 13.50$ & $20.06 \times 13.41$ & $19.41 \times 13.76$ & $20.06 \times 13.97$ & $20.94 \times 14.42$ \\
CT value $(\mathrm{HU})$ & $38.33^{*}$ & $25.59 \pm 8.27^{*}$ & $42.75 \pm 4.49$ & $37.5 \pm 3.77$ & $38.4 \pm 4.64$ & $37.6 \pm \sim 4.8$ \\
\hline
\end{tabular}

*, the data were based the former CT machine, so they were not comparable. TRS, transverse; MPR, multi-planar restructure; HU, Hounsfield unit.

should be considered if a reproductive or postmenopausal woman presents solitary or multiple pulmonary nodules in lung, when the patient has operation history of uterine leiomyoma. Even if the patient is asymptomatic. Long-term close radiological surveillance is required with either CT or MRI for patients with BML to monitor for early detection of recurrence or distant metastases $(13,14)$.

\section{Acknowledgments}

Funding: The study was supported by the National Natural Science Foundation of China (No. 81372299).

\section{Footnote}

Conflicts of Interest: The authors have completed the ICMJE uniform disclosure form (available at http://dx.doi. org/10.21037/tcr.2019.10.02). The authors have no conflicts of interest to declare.

Ethical Statement: The authors are accountable for all aspects of the work in ensuring that questions related to the accuracy or integrity of any part of the work are appropriately investigated and resolved. All procedures performed in studies involving human participants were in accordance with the ethical standards of the institutional and/or national research committee(s) and with the Helsinki Declaration (as revised in 2013). We received approval from the ethics committee of Jinan City People's Hospital. Written consent was obtained from the patient for the study and publication of this case report and accompanying images.

Open Access Statement: This is an Open Access article distributed in accordance with the Creative Commons Attribution-NonCommercial-NoDerivs 4.0 International License (CC BY-NC-ND 4.0), which permits the non- commercial replication and distribution of the article with the strict proviso that no changes or edits are made and the original work is properly cited (including links to both the formal publication through the relevant DOI and the license). See: https://creativecommons.org/licenses/by-nc-nd/4.0/.

\section{References}

1. Steiner PE. Metastasizing fibroleiomyoma of the uterus: report of a case and review of the literature. Am J Pathol 1939;15:89-07.

2. Abramson S, Gilkeson RC, Goldstein JD, et al. Benign metastasizing leiomyoma: clinical, imaging, and pathologic correlation. AJR Am J Roentgenol 2001;176:1409-13.

3. Tsunoda Y, Tanaka K, Hagiwara M. A case of benign metastasizing leiomyoma of the lung complicated with primary lung cancer. Jpn J Lung Cancer 2009;49:445-49.

4. Wolff M, Silva F, Kaye G. Pulmonary metastases (with admixed epithelial elements) from smooth muscle neoplasms. Report of nine cases, including three males. Am J Surg Pathol 1979;3:325-42.

5. Funakoshi Y, Sawabata N, Takeda S, et al. Pulmonary benign metastasizing leiomyoma from the uterus in a postmenopausal woman: report of a case. Surg Today 2004;34:55-7.

6. Rivera JA, Christopoulos S, Small D, et al. Hormonal manipulation of benign metastasizing leiomyomas: report of two cases and review of the literature. J Clin Endocrinol Metab 2004;89:3183-88.

7. Yoon G, Kim TJ, Sung CO, et al. Benign metastasizing leiomyoma with multiple lymph node metastasis: a case report. Cancer Res Treat 2011;43:131-33.

8. Fu Y, Li H, Tian B, et al. Pulmonary benign metastasizing leiomyoma: a case report and review of the literature. World J Surg Oncol 2012;10:268-72.

9. Efared B, Atsame-Ebang G, Sani R, et al. Unexpected pulmonary tumor: metastasis from a benign uterine 
leiomyoma in a post-menopausal woman: a case report. BMC Res Notes 2017;10:662-68.

10. Duncan SJ, Kadaria D. A Woman with a Lung Mass and Multiple Pulmonary Nodules. Am J Case Rep 2015;16:367-70.

11. Wei WT, Chen PC. Benign metastasizing leiomyoma of the lung: A case report and literature review. Oncol Lett 2015;10:307-12.

12. Miller J, Shoni M, Siegert C, et al. Benign Metastasizing Leiomyomas to the Lungs: An Institutional Case Series

Cite this article as: Li Q, Wei Y, Zhao X, Li C, Gao S, Chen B, Song S, Gao Q, Ma C, Li Q. A case of pulmonary benign metastasizing leiomyoma: clinicopathologic, immunohistochemical, and PET/CT analyses. Transl Cancer Res 2019;8(6):2496-2503. doi: 10.21037/tcr.2019.10.02 and a Review of the Recent Literature. Ann Thorac Surg 2016;101:253-58.

13. Raposo MI, Meireles C, Cardoso M, et al. Benign Metastasizing Leiomyoma of the Uterus: Rare Manifestation of a Frequent Pathology. Case Rep Obstet Gynecol 2018;2018:5067276.

14. Barber E, Eapen A, Mehta R, et al. Benign Metastasizing Leiomyoma to the Lung and Spine: A Case Report and Literature Review. Case Rep Oncol 2019;12:218-23. 